\title{
Successful Pregnancy Following Ectopic Pregnancy Treatment with Uterine Artery Embolization: A Case Report
}

\author{
Kristina Foley*, Beena Subba, Adewale Adeyemo \\ Department of Obstetrics and Gynaecology, North Middlesex University Hospital, UK
}

"Corresponding author: Foley Kristina, Department of Obstetrics and Gynaecology, North Middlesex University Hospital, UK. Email: Kristina.foley@nhs.net

Citation: Foley K, Subba B, Adeyemo A (2019) Successful Pregnancy Following Ectopic Pregnancy Treatment with Uterine Artery Embolization: A Case Report. Arch Surg Clin Case Rep 2: 111 DOI: 10.29011/ASCR-111/100111

Received Date: 17 April, 2019; Accepted Date: 13 May, 2019; Published Date: 22 May, 2019

Keywords: Cervical ectopic pregnancy, Ectopic pregnancy, Uterine artery embolization

\section{Introduction}

Cervical ectopic pregnancy is a rare form of ectopic pregnancy and occurs in less than $0.01 \%$ of pregnancies [1]. It occurs when a pregnancy implants itself into the cervical canal [1]. The aetiology of Cervical ectopic pregnancy is largely unknown, but possible risk factors include previous dilatation and curettage, Asherman's syndrome, previous caesarean section and IVF [2,3]. Cervical ectopic pregnancies are dangerous, due to possible erosion of the cervical blood vessels causing life threatening bleeding [4]. Due to the rare occurrence of cervical ectopic pregnancy, there is no nationalised recommendations or trials as to the most appropriate treatment option. Treatment options include Methotrexate IM [5], dilatation and curettage [6], hysterectomy, and since 1995, Uterine Artery Embolization (UAE) [7]. This case represents a successful outcome following treatment of cervical ectopic pregnancy treated with UAE. It was effective as a fertility-sparing method, as she had a further successful live term birth, with no complications such as miscarriage or premature ovarian insufficiency.

\section{Case}

A 43-year-old female Gradvia 2 Para $0+1$ presented for booking appointment in the antenatal clinic. She had a previous pregnancy resulting in a cervical ectopic 7 years prior, which was successfully treated with uterine artery emobilisation alone. She continued to attempt pregnancy and was found to have a septated uterus which was resected 3 years later. She then had a failed round of IVF, before becoming spontaneously pregnant at 43 years of age. She is known to have 1 large $>10 \mathrm{~cm}$ fibroid, for which she was admitted with fibroid degeneration during this pregnancy.

She was seen by consultant-led care throughout her pregnancy. She received Aspirin and Cyclogest in pregnancy and had monitoring cervical length and growth scans. Growth and dopplers remained normal throughout. She was given the option of mode of delivery and opted for an elective caesarean section. She was delivered at 38 weeks, and delivered a healthy male infant.

\section{Discussion}

As mentioned, cervical ectopic pregnancies are a rare form of ectopic pregnancy. Presenting complaint may include vaginal bleeding, with or without pain [3]. Diagnosis is made by ultrasound scan, where a gestational sac is seen in a widened cervical canal with an empty uterus [8]. Cervical ectopic on ultrasound can be confused with the cervical stage of miscarriage [3], and therefore diagnosis should be accompanied by doppler flow [9]. Before the routine use of USS, diagnosis used to often be made on dilatation and curettage for presumed incomplete miscarriage resulting in unexpected haemorrhage, sometimes leading to hysterectomy, stopping the woman's ability to have any future pregnancies [10].

Due to the low incidence rate of cervical ectopic pregnancies, there are no guidelines or standardised methods for most appropriate treatment. There are numerous treatment modalities that have been tried in practice. Methotrexate alone is minimally invasive and has been used at low gestation. It involves an Intramuscular (IM) injection that selectively targets cytotrophoblastic tissue of ectopic pregnancies while preserving fertility [5]. It is more successful at lower gestations, but there is no threshold value of when it is deemed effective [11]. Although it has low risk of cervical damage, as much as $60 \%$ of cases require additional procedures, such as curettage [8], and may have a prolonged recovery time following persistent bleeding. More invasive and with higher risks of complications are intra-amniotic injection and systemic uses of methotrexate [12].

After 12 weeks' gestation, a more invasive technique may be required such as surgical excision via curettage. This has a relatively high risk of haemorrhage, requiring blood transfusion 


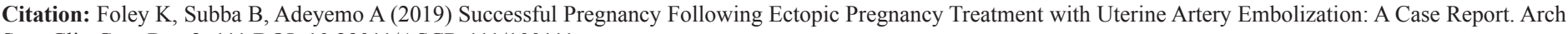
Surg Clin Case Rep 2: 111 DOI: 10.29011/ASCR-111/100111

and admission, and higher risk of cervical trauma [8]. Where there is irretractable bleeding, Foley's catheter insertion and emergency hysterectomy have been used as treatment methods [12]. Uterine artery embolization has been frequently used as treatment of cervical ectopic pregnancies, as stand-alone therapy or more commonly in conjunction with other methods. Benefits of UAE include effective control of excessive bleeding, and as a fertility-sparing method. There are multiple cases of successful live pregnancies following cervical ectopic treatment with UAE, however, some complications in future pregnancies has been reported, such as cervical adhesion and first trimester miscarriages [13].

There are some reported complications of UAE when used to manage uterine fibroids. These include problems in future pregnancies such as intra-uterine growth restriction and pre-term deliveries. However, many of these complications are difficult to differentiate from complications of pregnancy in a fibroid uterus [14]. Other reported complications are reduction in ovarian reserve, which is particularly important when considering this as a fertilitysparing option [14].

There are only a small handful of papers describing recurrent cervical pregnancies, so rates of recurrence with different treatment modalities are difficult to determine [15]. However, as cervical damage is a risk factor for cervical ectopic pregnancy, it might be assumed that further damage to the cervix with certain treatment modalities may increase the risk of recurrence of cervical ectopic pregnancy. Treatment modality will depend on gestation at diagnosis, age of patient, and need for fertility preservation, as well as the physician's technical capabilities. Treatment modalities can be combined to produce the best outcome for the patient.

\section{Conclusion}

This case gives an example of successful treatment of cervical ectopic pregnancy with UAE, and its use as a fertilitysparing method, allowing for successful subsequent pregnancy. There are no current guidelines on the best method of treatment for cervical ectopic pregnancy, and due to its low incidence rate, more case reports are needed to draw conclusions on the most appropriate treatment method as well as potential complications. This case gives argument for UAE as a relatively safe, effective, and fertility-sparing treatment method.

\section{Acknowledgement}

We would like to express our gratitude for the patient for allowing us to present her case

\section{References}

1. Ushakov FB, Elchalal U, Aceman PJ, Schenker JG (1997) Cervical pregnancy: past and future. Obstet Gynecol Surv 52: 45-59.

2. Shinagawa S, Nugayama M (1969) Cervical pregnancy as a possible sequela of induced abortion. Am J Obstet Gynecol 105: 282-284.

3. Gun M, Mavrogiorgis M (2002) Cervical ectopic pregnancy: a case report and literature review. Ultrasound Obstet Gynaecol 19: 297-301.

4. Samal SK, Rathod S (2015) Cervical ectopic pregnancy. J Nat Sci Biol Med 6: 257-260.

5. Pantelis A, Daniilidis A, Dinas K (2013) Conservative treatment of a 7-week cervical ectopic pregnancy after intra-uterine insemination. Hippokratia 17: 95.

6. Chang HC, Chiu TH, Hsieh TT, Lo LM (2015) Early cervical ectopic pregnancy: A case report and review of the literature. Journal of Medical Ultrasound 22: 117-119.

7. Takeda K, Mackay J, Watts S (2018) Successful management of cervical ectopic pregnancy with bilateral uterine artery embolization and methotrexate. Case reports in Emergency Medicine.

8. Pascual MA, Ruiz J, Tresserra F, Sanuy C, Grases PJ, et al. (2001) Cervical ectopic twin pregnancy: diagnosis and conservative treatment: Case Report. Human Reproduction 16: 585-586.

9. Jurkovic D, Hacket E, Campbell S (1996) Diagnosis and treatment of early cervical pregnancy: a review and report of two cases treated conservatively. Ultrasound Obstet. Gynecol 8: 373-380.

10. Leeman LM, et al. (2000) Cervical ectopic pregnancy. Arch Fam Med 9: $72-77$

11. The Practice Committee of the American Society for Reproductive Medicine. (2013) Medical treatment of ectopic pregnancy: a committee opinion. Fertility and Sterility 100: 638-644.

12. Singh S (2013) Diagnosis and management of cervical ectopic pregnancy. J Hum Reprod Sci 6: 273-276.

13. Hirakawa M, Tajima T, Yoshimitsu K, Irie H, Ishigami K, et al. (2009) Uterine Artery Embolization Along with the Administration of Methotrexate for Cervical Ectopic Pregnancy: Technical and Clinical Outcomes. American Journal of Roentgenol 192: 1601-1607.

14. Czuczwar P, Stępniak A, Wrona W, Woźniak S, Milart P, et al. (2016) The influence of uterine artery embolisation on ovarian reserve, fertility, and pregnancy outcomes-a review of literature. Prz Menopauzalny 15: $205-209$.

15. Radpour CJ, Keenan JA (2004) Consecutive cervical pregnancies. Fertility and Sterility 81: 210-213. 